\title{
The Challenge of T1 Contrast Agents for High-Magnetic Field MRI
}

\author{
Lothar Helm*
}

\begin{abstract}
Magnetic resonance imaging (MRI) is one of the most powerful diagnostic techniques used in clinics. The need for higher spatial resolution and better sensitivity led to the development of imagers working at high magnetic fields. The routine clinical use of $3 \mathrm{~T}$ MR systems raised the demand for MRI contrast agents working at this field or above. In the following we summarize the research in our research group on such high-field contrast agents.
\end{abstract}

Keywords: Contrast agent · Magnetic resonance imaging

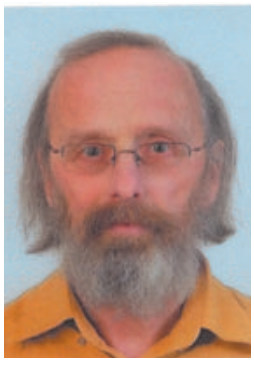

\section{Introduction}

Magnetic resonance imaging (MRI) became in the last decade one of the most powerful diagnostic techniques used in clinics. The need for higher spatial resolution and better sensitivity led to the development of $3 \mathrm{~T}$ imaging systems working at $127 \mathrm{MHz}^{[1,2]}$ which are now readily available in clinics. Several research institutions have now MRI instruments for human whole body examination working at magnetic fields of $7 \mathrm{~T}$ or even $9.4 \mathrm{~T}$. The increasing availability of high-field MRI systems $(3 \mathrm{~T})$ in clinics and the introduction of ultrahigh-field systems $(>3 \mathrm{~T})$ in research raise the demand for MRI contrast agents (CA) at high magnetic fields. ${ }^{[3]}$ In MRI the enhancement of image contrast by contrast media is not due to the visualization of the agent itself but due to changes in longitudinal $\left(T_{1}\right)$ or transverse $\left(T_{2}\right)$ relaxation of water proton spins induced by

${ }^{\star}$ Correspondence: Prof. Dr. L. Helm

Ecole polytechnique fédérale de Lausanne Institute of Chemical Sciences and Engineering EPFL-BCH

$\mathrm{CH}-1015$ Lausanne

Tel.: +41216939876

Fax: +41216939855

E-mail: lothar.helm@epfl.ch the presence of magnetic substances. ${ }^{[4]}$ In general, shortening of $T_{1}$ results in positive image contrast, meaning an increase of signal intensity caused by the presence of the agent. The shortening of $T_{2}$ results in a negative contrast which means that regions with short $T_{2}$ will appear dark in the image. Therefore, contrast agents can be classified in two categories: $T_{1}$ agents leading to a high relative change of longitudinal relaxation and $T_{2}$ agents which enhance much more transverse relaxation.

The contrast enhancement by paramagnetic chemical compounds is a function of the applied static magnetic field, $B_{0}$. Whereas transverse relaxation times have been reported to be only slightly dependent on the magnetic field for most organs, ${ }^{[2]}$ longitudinal relaxation times become markedly longer in a nonlinear way at high field. It is therefore difficult to predict if the contrast generated by administration of $T_{1}$-contrast agents increases or decreases by going to higher magnetic fields. ${ }^{[3]}$ Increasing a weak signal is much more attractive for contrast enhancement than decreasing an already feeble signal to zero.

Since the introduction of the first contrast agent for MRI in clinics, Magnevist ${ }^{\circledR}$, several gadolinium based compounds are in use:[5]

- extracellular contrast agents with a non-specific biodistribution: Dotarem $^{\circledR}$, Prohance $^{\circledR}$, Magnevist ${ }^{\circledR}$, Omniscan $^{\circledR}$, MultiHance ${ }^{\circledR}$,

- liver agents: Optimark ${ }^{\circledR}$ and Primovist $^{\circledR}$,

- agents for MR angiography: Vasovist ${ }^{\circledR}$ and Gadovist ${ }^{\circledR}$.

In the following I will first summarize very briefly which parameters influence the relaxivity of gadolinium-based contrast agents. Then I will present the clues how to optimize contrast agents for high and ultrahigh magnetic fields.

\section{Parameters Influencing the Efficiency of MRI Contrast Agents}

The enhancement of nuclear-spin relaxation due to the presence of paramagnetic centers has its source in the interaction between the observed nuclear-spin and the electron-spin of the paramagnetic. The unpaired electrons produce a magnetic field which fluctuates due to molecular motions and electron-spin relaxation. ${ }^{[6]}$ In MRI the main contribution to the detected signal arises from the nuclear spin of the water protons and not from fat or protein protons. In the theoretical description of relaxation enhancement two types of water molecules are distinguished (Fig. 1):

- coordinated water molecules directly bound to the paramagnetic ion,

- bulk water molecules diffusing freely in the vicinity of the paramagnetic complex.

The relaxation enhancement generated by the presence of a $1 \mathrm{mM}$ concentration of gadolinium ions, called relaxivity $\left(r_{1}\right)$, is therefore described by an inner-sphere $\left(r_{1}{ }^{\text {S }}\right)$ and an outer-sphere $\left(r_{1}{ }^{\text {os }}\right)$ contribution. ${ }^{77]}$ The outer-sphere contribution depends on the closest distance of approach

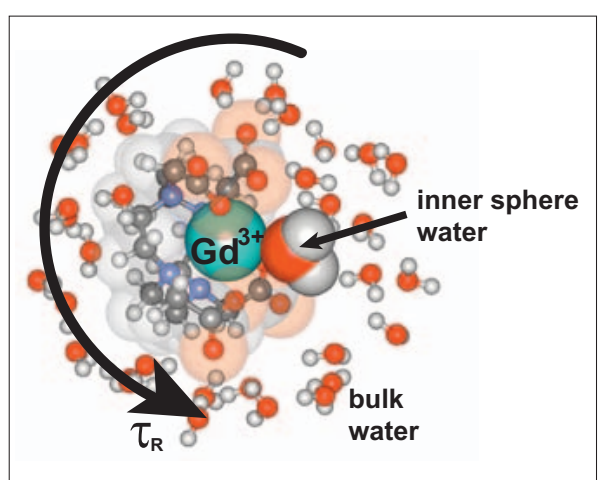

Fig. 1. Representation of a $\mathrm{Gd}^{3+}$ complex with inner sphere and bulk water molecules. 
between the electron- and the nuclear spin and on the mutual translational diffusion between water molecules and the gadolinium complex. Both cannot be altered significantly by creating new compounds. The development of more efficient $\mathrm{T}_{1}$-contrast agent is therefore based on the increase of the inner-sphere relaxivity.

Details of the theoretical description of paramagnetic relaxation enhancement can be found in recent reviews. ${ }^{[4,8,9]}$ The most important parameters and their influence on relaxivity are:

- the rotational correlation time $\left(\tau_{R}\right)$; longer $\tau_{R}$, as obtained by larger compounds, leads to a marked increase of $r_{1}$ Is at magnetic fields usual for MRI;

- depending on the magnetic field an optimal exchange rate constant $\left(k_{\mathrm{ex}}\right)$ for water exchange from the $1^{\text {st }}$ coordination sphere of the Gd-complex exists;

- the electron spin relaxation should be as slow as possible;

- the number of water molecules $(q)$ which is $q=1$ for all commercial agents should be increased without deterioration of the excellent stability of the complexes.

\section{Towards Efficient Contrast Agents for High Magnetic Fields}

At a common MRI magnetic field of $1.5 \mathrm{~T}$ relaxivities of the order of $5 \mathrm{mM}^{-1}$ $\mathrm{s}^{-1}$ are achieved with actual commercial contrast agents. By optimizing $\tau_{\mathrm{R}}, k_{\mathrm{ex}}$ and electron spin relaxation relaxivities of more than $50 \mathrm{mM}^{-1} \mathrm{~s}^{-1}$ can be obtained with compounds with $q=1$. These high values reduce markedly at high magnetic field of $3 \mathrm{~T}$ and above. At fields above $3 \mathrm{~T}$ highest relaxivities are no longer obtained with large compounds having rotational correlation times well above $1 \mathrm{~ns}$ but by mid-size compounds with $500 \mathrm{ps}<\tau_{\mathrm{R}}<$ 1000 ps (Fig. 2).

Actually there are only two strategies to increase the efficiency of gadoliniumbased contrast agents at ultra-high magnetic fields:

- increase of the number of first coordination sphere water molecules to $q=2$ or $q=3$;

- construction of compounds or particles comprising several Gd-ions.

To increase the number of inner-sphere water molecules the number of binding atoms of the chelating ligand has to be reduced. Because $\mathrm{Gd}^{3+}$ has a maximum coordination number of nine chelating ligands with seven or less binding atoms have to be synthesized. This leads ineluctably to a decrease of the thermodynamic stability of the complex. If the residence time of the contrast agent in the living organism is not too long relatively safe compounds

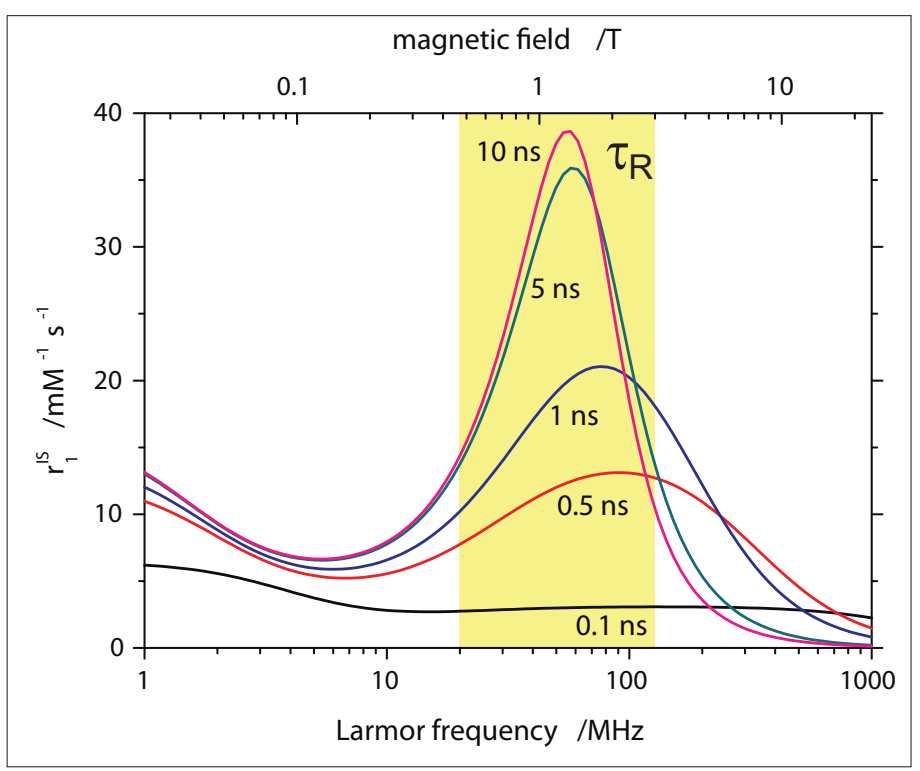

Fig. 2. Magnetic field dependence of inner sphere relaxivity $r_{1}^{\text {IS }}$ for different rotational correlation times $\tau_{\mathrm{R}}$. Magnetic fields common for MRI are in yellow.

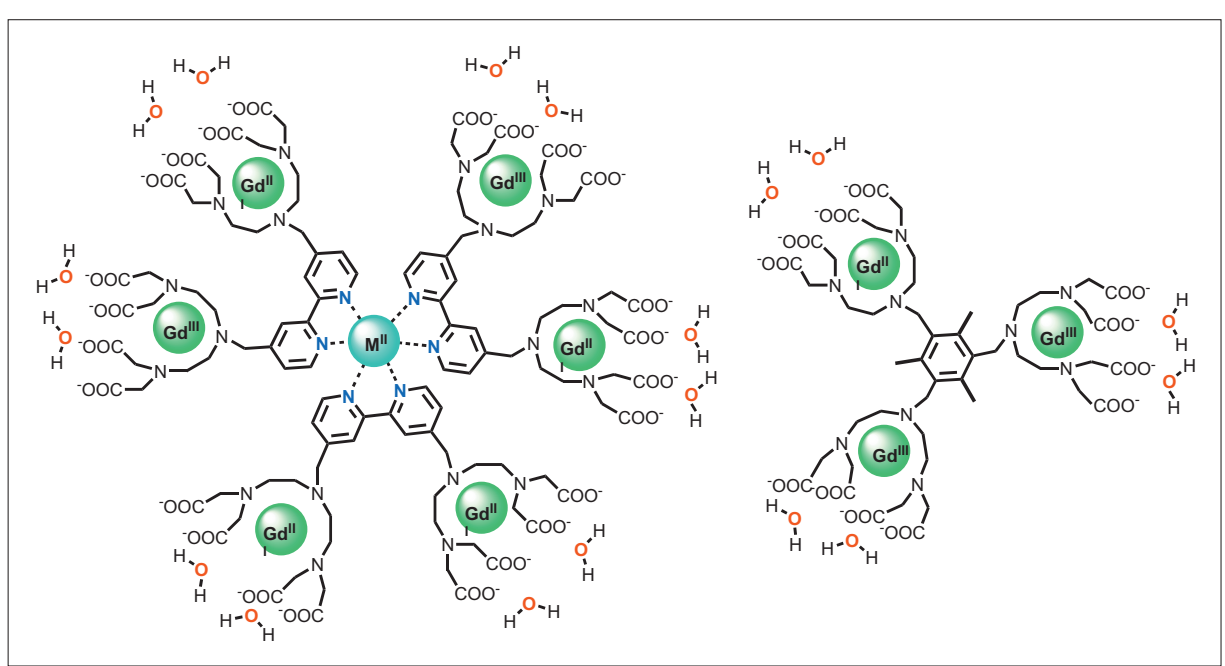

Fig. 3. Mid-size molecules with with six (metallostar $\left\{\mathrm{M}\left[\mathrm{Gd}_{2} \mathrm{bpy}-\mathrm{DTTA} \mathrm{A}_{2}\left(\mathrm{H}_{2} \mathrm{O}\right)_{4}\right]_{3}\right\}^{4-}, \mathrm{M}=\mathrm{Fe}^{2+}, \mathrm{Ru}^{2+}$, left) and three (trimethylbenzene complex) $\mathrm{Gd}^{3+}$ centers (right).

are achieved by the use of kinetically stable (inert) complexes. ${ }^{[10]}$ The transmetalation reaction (replacement of $\mathrm{Gd}^{3+}$ by an endogenous ion like $\mathrm{Ca}^{2+}, \mathrm{Zn}^{2+}$ or $\mathrm{Fe}^{3+}$ ) has to be slow compared to the elimination from the body. Two examples of possible chelating ligands can be found in ref. [9].

The construction of compounds comprising several Gd-ions is limited by the maximum size of the entity: remember that the rotational correlation time should be below $1 \mathrm{~ns}$ ! This correlation time can be estimated by the Debye-Stokes-Einstein relation which relates the radius of the particle $(a)$, respectively the volume $(V)$, and the viscosity $(\eta)$ to $\tau_{\mathrm{R}}$.

$$
\tau_{R}=\frac{4 \pi a^{3} \eta}{3 k T}=V \frac{\eta}{k T}
$$

From this relation we can estimate that the compounds should have a radius small- er than $1 \mathrm{~nm}$. What is desirable is a 'high density of relaxation enhancement', which we can define as the relaxation enhancement divided by the volume of the compound approximated by a sphere. Several attempts have been made in our laboratory to synthesize such units (Fig. 3). ${ }^{[1-13]}$ The most prominent is certainly the "metallostar' which has six $\mathrm{Gd}^{3+}$ ions assembled around a divalent iron- or ruthenium ion. In another example three Gd-complexes are linked to a benzene core. In calculating the relaxivity of these compounds we have to account not only for the global rotation of the entity but also for internal rotation of the bound water molecule in the first coordination sphere or of the whole chelating group.

Another approach is to increase the longitudinal relaxation rate locally which consists in attaching many gadolinium ions to one particle. Even if the relaxivity of each Gd-chelate is moderate at high 


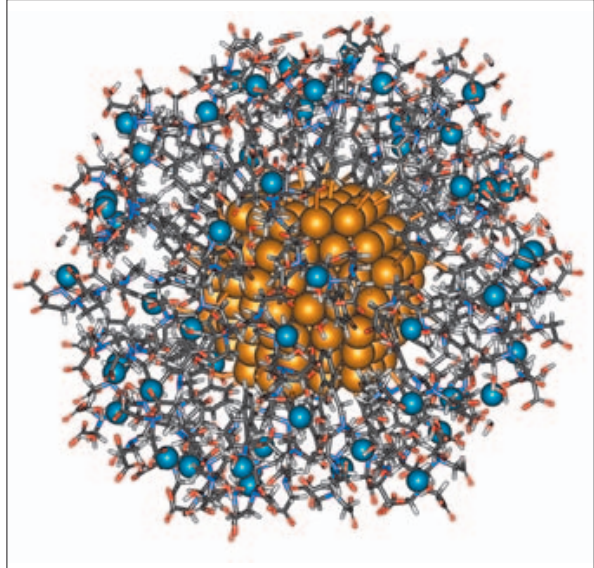

Fig. 4. Model of a gold nanoparticle bearing 56 covanlently bound $\left[\mathrm{Gd}(\mathrm{DTTA})\left(\mathrm{H}_{2} \mathrm{O}\right)_{2}\right]^{-}$chelating units.

magnetic field, a big relaxation enhancement can be achieved if we consider the whole particle. An example for such a unit is a gold nanoparticle (Fig. 4). ${ }^{[14]}$ Such a particle bearing about $50 \mathrm{Gd}^{3+}$ ions at the surface increases the $T_{1}$ relaxation of water protons by $500 \mathrm{~s}^{-1}$ at $9.4 \mathrm{~T}$ (calculated for a $1 \mathrm{mM}$ concentration of particles). The mean diameter of the particles is about 5 $\mathrm{nm}$ making it small enough to leave the blood vessels.

Even larger constructs can be obtained by confining gadolinium-based contrast agents in nanoporous microfabricated silicon particles (SiMPs, Fig. 5). ${ }^{[15]}$ The size, shape and surface properties of the SiMPs can be rationally designed and tailored to enhance the accumulation at biological target sites. The nanoconstructs may potentially be used for single-cell imaging techniques, where high relaxivity and large local concentrations $\left(\left[\mathrm{Gd}^{3+}\right]>10^{7} /\right.$ cell $)$ are needed. Finally these nanoconstructs might also be loaded with multiple agents, such as other nanoparticles and/or small molecules and drugs, to generate multifunctional systems that have both imaging and therapeutic capabilities.

\section{Acknowledgements}

The author thanks the Swiss National Science Foundation for financial support. This research was carried out in the frame of the EC COST Action D-38 'Metal-Based Systems for Molecular Imaging Applications'.

Received: June 6, 2011

[1] K. Ugurbil, G. Adriany, P. Andersen, W. Chen, M. Garwood, R. Gruetter, P.-G. Henry, S.-G. Kim, H. Lieu, I. Tkac, T. Vaughan, P.-F. Van De Moortele, E. Yacoub, X.-H. Zhu, Magn. Reson. Imag. 2003, 21, 1263.

[2] F. Schick, Eur. Radiol. 2005, 15, 946.

[3] S. Trattnig, K. Pinker, A. Ba-Ssalamah, I.-M. Nöbauer-Huhmann, Eur. Radiol. 2006, 16, 1280.

[4] P. Caravan, in 'Molecular and Cellular MR Imaging', Eds. M M. J. Modo, J. W. M. Bulte, CRC Press LLC, Boca Raton, 2007, pp 13-26.
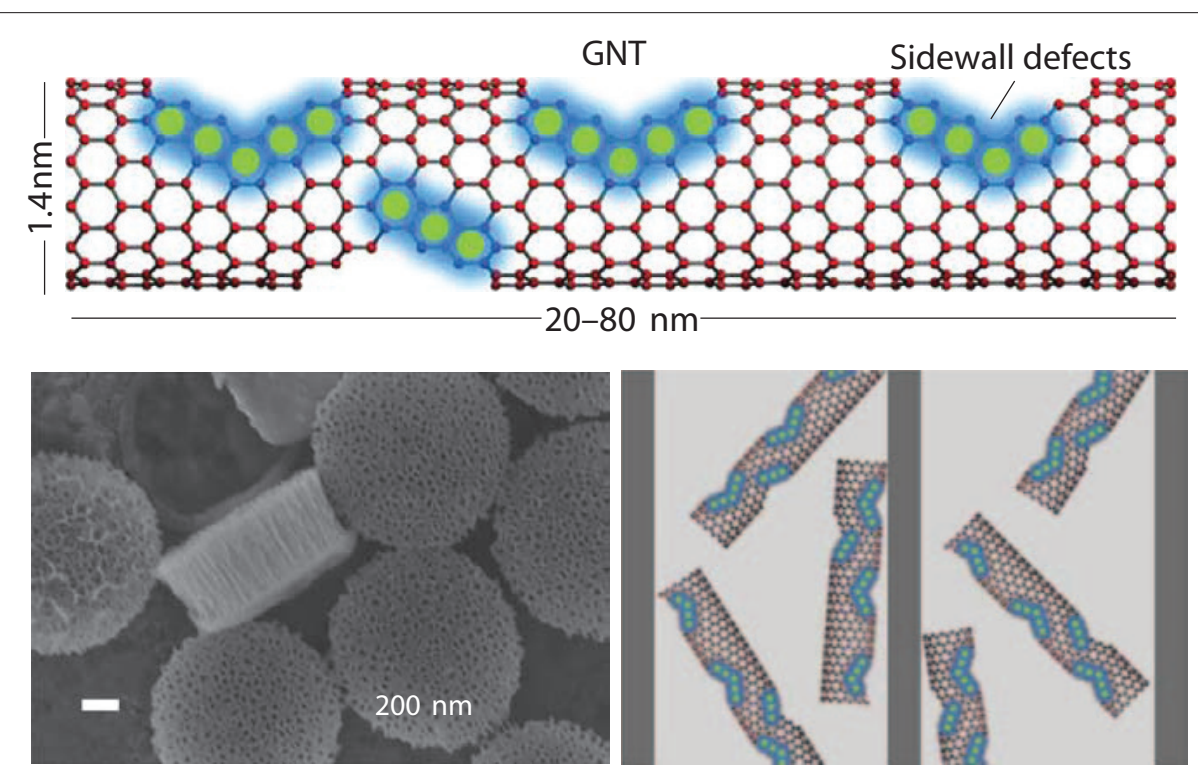

Fig. 5. Top: Ultra-short single walled carbon nanotube loaded with $\mathrm{Gd}^{3+}$ ions (GNT). Bottom left: Scanning electron micrographs of quasi-hemispherical particles (H-SiMP: diameter, $1.6 \mu \mathrm{m}$; thickness, $\sim 0.6 \mu \mathrm{m}$ ). Bottom right: Schematic drawing showing GNTs entrapped within the porous structure of the SiMPs.

[5] M.-F. Bellin, Eur. J. Radiol. 2006, 60, 314.

[6] J. Kowalewski, L. Mäler, 'Nuclear Spin Relaxation in Liquids: Theory, Experiments, and Applications', Taylor \& Francis, New York 2006.

[7] L. Helm, Prog. in NMR Spect. 2006, 49, 45.

[8] P. Caravan, C. T. Farrar, L. Frullano, R. Uppal, Contrast Med. Mol. Imaging 2009, 4, 89.

[9] L. Helm, Future Med. Chem. 2010, 2, 385.

[10] E. Brücher, A. D. Sherry, in 'The Chemistry of Contrast Agents in Medical Magnetic Resonance Imaging', Eds. A. E. Merbach, É. Tóth, John Wiley \& Sons Ltd, Chichester, 2001, pp 243-279.

11] L. Moriggi, A. Aebischer, C. Cannizzo, A. Sour, A. Borel, J.-C. G. Bünzli, L. Helm, Dalton Trans. 2009, 2088.
[12] J. B. Livramento, C. Weidensteiner, M. I. M. Prata, P. R. Allegrini, C. F. G. C. Geraldes, L. Helm, R. Kneuer, A. E. Merbach, A. C. Santos, P. Schmidt, É. Tóth, Contrast Med. Mol. Imaging 2006, 1, 30.

[13] J. B. Livramento, L. Helm, A. Sour, C. O'Neil, A. E. Merbach, É. Tóth, Dalton Trans. 2008 1195

[14] L. Moriggi, C. Cannizzo, E. Dumas, C. R. Mayer, A. Ulianov, L. Helm, J. Am. Chem. Soc. 2009, 131, 10828.

[15] J. S. Ananta, B. Godin, R. Sethi, L. Moriggi, X. Liu, R. E. Serda, R. Krishnamurthy, R. Muthupillai, R. D. Bolskar, L. Helm, M. Ferrari, L. J. Wilson, P. Decuzzi, Nat. Nanotechnol. 2010, 5,815 . 